\title{
QUANTIFICAÇÃO POR DRX DOS COMPONENTES CARBONOSOS PRESENTES NO PÓ DE BALÃO DO ALTO-FORNO
}

André da Silveira Machado ' André Sampaio Mexias ${ }^{2}$ Antônio Cezar Faria Vilela ${ }^{3}$ Eduardo Osório ${ }^{4}$

\section{Resumo}

A difração de raios $X(D R X)$ é uma técnica padrão para caracterizar a estrutura cristalina dos materiais. Ela também pode ser utilizada para quantificar o tamanho dos cristalitos $\left(L_{c}\right)$ dos materiais carbonosos. Este trabalho tem por objetivos identificar e quantificar os componentes carbonosos (char e finos de coque) presentes no pó de balão do Alto-forno (AF) pelas técnicas de DRX e de análise elementar de carbono. As partículas coletadas no pó de balão do AF, compostas por char, finos de coque e outros, foram fracionadas e analisadas quimicamente. Posteriormente, as frações foram moídas, desmineralizadas e analisadas por DRX. Amostras de char produzidas em laboratório e coque foram utilizadas como padrões para a quantificação. A técnica utilizada revela as diferenças na dimensão dos cristalitos destes materiais carbonosos, que a estrutura do coque é mais ordenada que a estrutura do char. Com base nessas diferenças podem-se quantificar as proporções de char e finos de coque no pó de balão do AF. A DRX permite quantificar, para o pó de balão em estudo, que $93 \%$ da matéria carbonosa se encontra na forma de coque e 7\%, na forma de char.

Palavras-chave: Char; Coque; Difração de raios X; Injeção de carvão pulverizado.

\section{QUANTIFICATION OF COAL CHAR AND COKE FINES IN THE OFF-GAS BLAST FURNACE SAMPLES BY X-RAY DIFFRACTION}

\begin{abstract}
X-ray diffraction $(X R D)$ is a standard technique of characterizing structure of materials. The aim of this work is to identify and quantify carbonaceous components (coal, char and coke fines) in the off-gas blast furnace (BF) through the use of the XRD technique and ultimate analysis. The dust collected in the off-gas BF, which contains char, coke fines, metallic oxides, etc., was fractioned and chemically analyzed. In addition, the fractions were grounded, demineralized and analyzed by ultimate analysis and XRD. This technique reveals differences in crystallite size of the carbonaceous materials. The coke structure shows to be more ordered (bigger $L_{c}$ ) than the char structure. Based on these differences, it is possible to quantify the fines proportions of char and coke in the blast furnace flue dust. The estimated char proportion in the carbonaceous material of the flue dust is $7 \%$.
\end{abstract}

Key words: Char; Coke; X-ray diffraction; Pulverized coal injection.

\section{INTRODUÇÃO}

O alto-forno (AF) é a principal tecnologia de redução no mundo empregada para a produção de aço. Este processo tem sofrido modificações por meio de melhorias que foram implantadas ao longo dos anos. A injeção de carvão pulverizado (Pulverized Coal Injection -
$\mathrm{PCl})$ nos AFs é uma tecnologia importante adotada pelas usinas siderúrgicas para diminuir o consumo de coque e aumentar a produtividade dos AFs.(I) Nas últimas décadas, as taxas de injeção de carvão pulverizado têm aumentado na maior parte dos AFs, alcançando valores entre $150-220 \mathrm{~kg} /$ tgusa (quilograma por tonelada de ferro gusa).(2)

\footnotetext{
'Físico, M.Sc., Bolsista, Laboratório de Siderurgia, Universidade Federal do Rio Grande do Sul - UFRGS, Av. Bento Gonçalves, 9500, Cep 9I50I-970, Porto Alegre, RS, Brasil. E-mail: andre.machado@ufrgs.br

${ }^{2}$ Geólogo, Dr., Professor, Instituto de Geociências, Universidade Federal do Rio Grande do Sul - UFRGS, Av. Bento Gonçalves, 9500, Cep 9I50I-970, Porto Alegre, RS, Brasil.E-mail: andre.mexias@ufrgs.br

${ }^{3}$ Engenheiro Metalúrgico, Dr., Professor, Laboratório de Siderurgia, Universidade Federal do Rio Grande do Sul - UFRGS, Av. Bento Gonçalves, 9500 , Cep 9150I-970, Porto Alegre, RS, Brasil. E-mail: vilela@ufrgs.br

${ }^{4}$ Engenheiro Metalúrgico, Dr., Professor, Laboratório de Siderurgia, Universidade Federal do Rio Grande do Sul - UFRGS, Av. Bento Gonçalves, 9500 , Cep91501-970, Porto Alegre, RS, Brasil.E-mail: eosorio@ufrgs.br
} 
O principal problema associado à utilização de altas taxas de $\mathrm{PCl}$ é a geração de char (carvão incombusto) na zona de combustão. Esse material percorre um caminho ascendente junto ao fluxo gasoso, podendo prejudicar a permeabilidade da carga do AF. A presença de altos teores de char, nas amostras sólidas recolhidas do gás de saída do $A F$, é resultado da injeção de um carvão pulverizado com uma combustibilidade inadequada e de uma operação instável do AF. Portanto, conhecer a proporção de char que sai do $\mathrm{AF}$ poderá contribuir na seleção dos carvões utilizados e na otimização da prática de $\mathrm{PCl}^{(3)}$

Os particulados contidos nos gases de saída do $\mathrm{AF}$ são coletados no sistema de limpeza, denominado balão (pó de balão), e são constituídos basicamente de óxidos metálicos e materiais carbonosos. A fração carbonosa é oriunda de finos de coque, char e, em alguns casos, carvão não reagido, sendo que o teor de carbono elementar pode atingir valores de até $70 \% .^{(4,5)}$

A identificação das partículas carbonosas encontradas no pó do AF não é trivial. As partículas de char podem representar uma pequena fração do pó e ainda estarem misturadas a grandes quantidades de finos de coque. A técnica de microscopia óptica já é utilizada para o estudo e a quantificação das partículas carbonosas do pó de $A F^{(5-7)}$ Contudo, o uso exclusivo do microscópio óptico para identificar as partículas carbonosas de char e finos de coque pode levar a resultados ambíguos. A principal dificuldade para uma análise quantitativa dessas amostras está relacionada ao pequeno tamanho das partículas e à possibilidade de algumas delas estarem recobertas com cinza e/ou álcalis e cloretos precipitados. Esta limitação imposta à técnica de microscopia ótica não se aplica à técnica de DRX. Seu feixe altamente energético pode penetrar nas partículas e identificá-las, mesmo se estiverem mascaradas por cinzas. ${ }^{(3)}$ Por meio desta técnica pode-se distinguir claramente o char do coque, uma vez que estes materiais apresentam diferentes graus de ordenamento em suas estruturas carbonosas. ${ }^{(8)} \mathrm{A}$ DRX, associada à medida do teor carbonoso, pode ser utilizada como procedimento padrão para determinar o teor de char no pó de balão. Tal conhecimento pode servir como um indicativo para monitorar o desempenho do $\mathrm{PCl}$ no AF. Essa técnica também poderá ser empregada para identificar diferentes tipos de char e com isso estudar a eficiência de combustão de distintos carvões, em misturas para PCl. Outra possibilidade seria avaliar os mecanismos de geração e consumo de finos de coque no $A F^{(9)}$

Este trabalho tem por objetivo identificar e quantificar os componentes carbonosos (char e finos de coque) presentes no pó de balão do $\mathrm{AF}$ por meio das técnicas de DRX e de análise elementar de carbono.

\section{MATERIAL E MÉTODOS}

A metodologia empregada para obter a quantificação dos materiais carbonosos presentes no pó de balão abrange: I) caracterizar química e granulométrica as amostras de pó de balão, carvões e coque utilizadas no processo; 2) obter uma amostra de char produzida em simulador da zona de combustão a partir dos carvões estudados; 3) realizar análise por DRX de todas as amostras e frações do pó de balão; 4) analisar os difratogramas para o cálculo do $L_{c}$.

\section{I Matérias Primas e Caracterização da Matéria Carbonosa}

Neste trabalho utilizaram-se amostras de carvões ( $C A$ e $C B$ ), de char $(C h A B)$, de coque (CQ) e uma amostra de pó de balão $(\mathrm{PB})$ recolhido de um $\mathrm{AF}$ a coque com $\mathrm{PCl}$. O char foi produzido num simulador da zona de combustão. Esse equipamento possui dois fornos: um para o pré-aquecimento do oxigênio até a temperatura de sopro no $\mathrm{AF}\left(1.200^{\circ} \mathrm{C}\right)$ e outro para simular as condições térmicas na zona de combustão $\left(1.600^{\circ} \mathrm{C}\right) .^{(10)} \mathrm{A}$ amostra de char utilizada foi produzida em atmosfera inerte de $\mathrm{N}_{2}$ a fim de prover material suficiente para as análises. $O$ char $\mathrm{ChAB}$ foi obtido de uma mistura dos carvões $\mathrm{CA}$ e $\mathrm{CB}$ (CAB) utilizada no PCl.

As amostras de carvão, $\mathrm{CQ}$ e $\mathrm{PB}$, foram moídas (norma ASTM D20I3), (11) para a retirada de frações e realizadas as respectivas análises imediata (norma ASTM D5I42) (12) e elementar (analisador de CHNS, normas ASTM D5373(13) e ASTM D4239(14)).

A amostra de pó PB foi fracionada em sete faixas granulométricas. ${ }^{(4)}$ Essa separação foi empregada para verificar como as partículas carbonosas se distribuem no pó e identificar as possíveis frações compostas unicamente por char e coque. O teor de carbono das frações do pó foi analisado em analisador de carbono (ASTM 5373).(13)

\subsection{Difração de Raios X}

\subsection{Caracterização das estruturas carbonosas}

As análises químicas convencionais, imediata e elementar, não conseguem distinguir os diferentes tipos de estruturas carbonosas e suas proporções presentes nas amostras de pó de balão. Pode-se considerar que os materiais carbonosos possuem dois tipos de estruturas: carbonos na forma cristalina (grafítico) ou amorfa. ${ }^{(15)}$ Com o aumento do rank do carvão, são empilhadas mais camadas carbonosas. Diferentes carvões possuem diferentes proporções de carbonos cristalinos e amorfos, o que pode estar associado a sua reatividade. Os chars apresentam estrutura cristalina um pouco mais organizada em relação aos seus carvões de origem..$^{(9)}$ Lu et al. ${ }^{(15)}$ apresentam um estudo quantitativo detalhado via DRX das estruturas carbonosas dos carvões.

A DRX é uma técnica não destrutiva, amplamente estabelecida para medir com boa reprodutibilidade os parâmetros estruturais dos materiais carbonosos. $\mathrm{O}$ valor 
de $L_{c}$ representa a dimensão média estatística de bilhões de cristalitos na amostra, em nível nanométrico. Materiais carbonosos, como carvões, char e coque, podem ser diferenciados a partir das dimensões de seus cristalitos. Quanto mais ordenada for a estrutura carbonosa maior será a dimensão dos cristalitos $\left(L_{c}\right)$. A cristalinidade de uma estrutura carbonosa pode ser identificada pela forma do pico (002) do carbono. Picos estreitos representam grande grau de ordenamento estrutural. $O$ perfil do pico (002) do coque apresenta-se mais agudo que o do char (maior $L_{c}$ ), portanto, sua estrutura cristalina possui maior organização. $^{(3)}$

A técnica de DRX foi utilizada para determinar a dimensão dos cristalitos $\left(L_{c}\right)$ da matéria carbonosa presente nas amostras estudadas. As frações do PB, carvões e o $\mathrm{CQ}$, foram previamente desmineralizadas para evitar o efeito da matéria mineral na análise quantitativa via $\mathrm{DRX}$. A amostra de char não foi desmineralizada em função da pequena quantidade de amostra. A desmineralização foi realizada conforme a descrição dada em Lu et al.(15)

As análises de DRX foram realizadas em um difratômetro SIEMENS modelo D 500, utilizando um tubo de radiação $\mathrm{Cu}-\mathrm{K} \alpha$, (40 kV, $25 \mathrm{~mA})$. As amostras foram analisadas em ângulos de varredura de $10-40^{\circ}(2 \theta)$, num passo de $0,05^{\circ}$, com intensidades registradas por $4 \mathrm{~s}$ em cada passo. As curvas obtidas nas difrações foram ajustadas, segundo um perfil do tipo gaussiano, utilizando o programa OriginPro 7.5. Foi utilizada a curva split Pearson VII para o ajuste assimétrico do pico (002) do carbono e calculados os valores de $L_{c}$ a partir destes dados.

O alargamento de pico devido ao instrumental foi calibrado com um padrão de hexaboreto de lantânio $\left(\mathrm{LaB}_{6}\right)$, de acordo com a Equação I, dada por Warren ${ }^{(16)}$ :

$$
\beta=\sqrt{B^{2}-B_{0}^{2}}
$$

onde $B$ é a largura total a meia-altura da intensidade máxima (full widht at half maximum - FWHM) do pico (002) do carbono e $B$ é a FWHM do pico (3I I) do $\mathrm{LaB}_{6}$, sendo este o mais próximo ao pico (002) do carbono. $O$ valor de $L_{c}$ foi calculado utilizando a Equação 2 de Scherrer citada por Warren ${ }^{(16)}$

$$
L c=\frac{0,89 \lambda}{\beta \cdot \operatorname{Cos}(\theta)}
$$

onde: $L_{c}$ é altura do cristalito (dimensão do empilhamento das camadas do retículo), em ângstron; $\lambda$ é comprimento de onda dos raios $X$ incidentes (neste caso, o valor de $\lambda$ do Cu-K $\alpha_{1}(\mathrm{I}, 54060 \AA)$ ); $\beta$ é a FWHM corrigida do pico (002), em radianos e $\theta$ é o ângulo de Bragg do pico (002), em graus.

\subsubsection{Análise quantitativa por DRX}

A quantificação do teor de char e finos de coque na amostra de PB foi obtida a partir do teor carbonoso das frações do PB e de uma interpolação matemática $\left(\%\right.$ Char $\left.\times L_{c}\right)$, gerada a partir do $L_{c}$ dos padrões de char e coque.

O teor carbonoso das frações do PB foi calculado levando-se em conta o teor de carbono e a distribuição granulométrica do $\mathrm{PB}$. $\mathrm{O}$ padrão de char $\mathrm{ChAB}$ foi obtido num simulador da zona de combustão. Para o padrão de coque $\mathrm{CQ}_{\mathrm{PB}}$ é utilizada a fração 250 - $425 \mu \mathrm{m}$ do $\mathrm{PB}$, de acordo com a literatura. ${ }^{(4)}$

A interpolação linear (Equação 3) pode ser construída com os valores de $L_{c}$ das amostras $100 \%$ ChAB $(\mathrm{LCCH})$ e $100 \% \mathrm{CQ}_{\mathrm{PB}}(\mathrm{LcCQ})$ :

$$
\% \mathrm{Ch}=\mathrm{aL}_{\mathrm{c}}+\mathrm{b}
$$

onde: $L_{c}$ é o tamanho de cristalito médio da fração do PB analisado; \%Ch é o respectivo teor de char; $a=-100 /\left(L_{c C Q}-L_{c C H}\right) ; b=100 L_{c c Q} /\left(L_{c C Q}-L_{c C H}\right)$.

Relacionando-se, para cada fração do $\mathrm{PB}$, o teor carbonoso, o $L_{c}$ da respectiva faixa e a Equação 3, calculam-se os teores de char e coque nos PBs estudados.

\section{RESULTADOS E DISCUSSÃO}

\section{I Caracterização das Amostras}

Os resultados das análises imediata e elementar para os carvões e o $\mathrm{CQ}$ são mostrados na Tabela I. Esta tabela também apresenta o teor de matéria inorgânica e carbono para o PB.

Os carvões $C A$ e $C B$ têm médio e baixo teores de matéria volátil, respectivamente, teores de cinza próximos aos $10 \%$ e alto teor de carbono elementar, em torno de $76 \%$. O CQ apresenta teor de cinza semelhante ao dos carvões $(9,81 \%)$ e alto teor de carbono elementar

\begin{tabular}{|c|c|c|c|c|}
\hline $\begin{array}{c}\text { Análise imediata } \\
\text { (\%bs) }\end{array}$ & CA & CB & CQ & PB \\
\hline Matéria volátil & 24,46 & 14,42 & 1,66 & Matéria inorgânica \\
\hline Cinzas & 9,43 & II,34 & 9,81 & (\%bs) \\
\hline Carbono fixo & 66,11 & 74,24 & 88,53 & 57,06 \\
\hline \multicolumn{5}{|c|}{ Análise elementar (\%bs) } \\
\hline Carbono & 75,92 & 76,84 & 86,76 & Carbono (\%bs) \\
\hline Hidrogênio & 6,26 & 5,60 & 0,34 & 43,57 \\
\hline Nitrogênio & $\mathrm{I}, 72$ & 1,64 & $\mathrm{I}, 08$ & \\
\hline Enxofre & 0,53 & $0,7 \mathrm{I}$ & 0,88 & \\
\hline Oxigênio & 6,14 & 3,87 & 1,13 & \\
\hline
\end{tabular}
$(86,76 \%)$. O teor de carbono do PB fica em torno de $44 \%$

Tabela I. Análises químicas dos carvões, coque e PB 
e o seu teor de matéria inorgânica, de cerca de $57 \%$. No trabalho de Sahajwalla e Gupta ${ }^{(9)}$ são encontrados teores de carbono inferiores a $50 \%$, para amostras de pó de balão. Segundo o autor, esses valores podem chegar à ordem de $70 \%$.

Os teores carbonosos das frações granulométricas do PB são apresentados na Figura I.

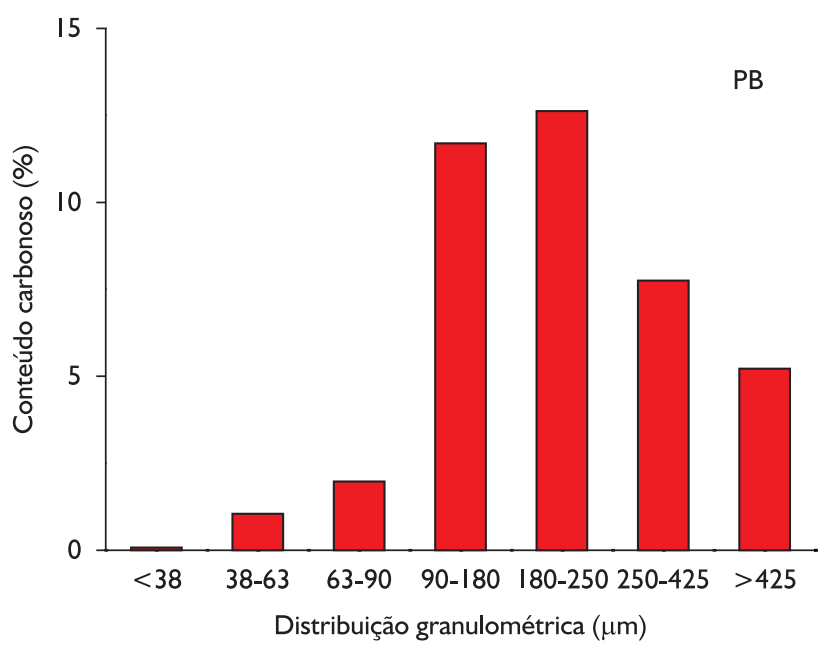

Figura I. Carbono total por faixa granulométrica do PB.
O teor carbonoso é calculado levando em conta - teor de carbono (análise elementar) e a quantidade de amostra por fração do PB. O carbono presente nas frações do PB é considerado oriundo de finos de coque, char incombusto e, em alguns casos, carvão não reagido. ${ }^{(5,7)}$ Observa-se, na Figura I, que as frações de maior granulometria apresentam os maiores teores de carbono, sobretudo na faixa de $90-425 \mu \mathrm{m}$. A fração inferior a $38 \mu \mathrm{m}$ apresenta teor insignificante de carbono. O teor carbonoso nas frações é inferior a 13\%. Resultados semelhantes são encontrados por Sahajwalla e Gupta..$^{(9)}$

\subsection{Difração de Raios X}

\subsection{Tamanho de cristalito - organização cristalina do carbono}

O char e o coque são as duas principais formas carbonosas presentes nas amostras de pó de balão do AF. A Figura 2 apresenta os espectros de raios $X$, perfis do pico (002) do carbono e valores de $L_{c}$ para as amostras de carvão, char, $C Q, C Q_{P B}$ e $P B$.

Pode-se notar, na Figura 2, que as amostras apresentam distintos perfis para o pico (002) do carbono e que o pico do coque é relativamente mais agudo do que
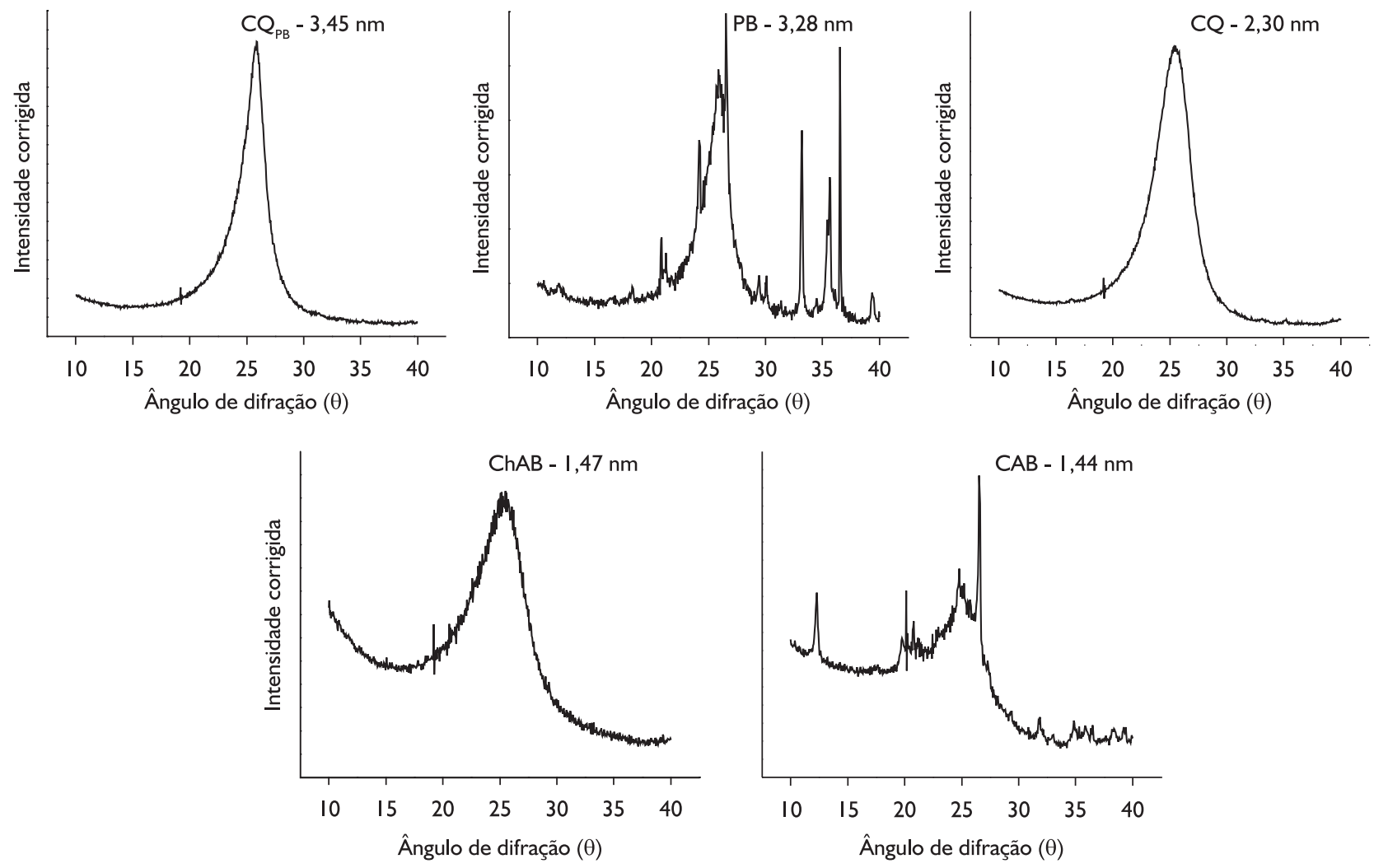

Figura 2. Espectros de difração de raios $X$ e perfis do pico (002) do carbono para as amostras $C Q_{P B}, P B, C Q, C h a r, C A B$ e valores de $L_{c}$ $\left(\mathrm{Cu}-\mathrm{K} \alpha_{1}\right)$. 
o do char. A distribuição das amostras, em ordem crescente dos valores de $L_{c}$, é a seguinte: $C A B-\mathrm{I}, 44 \mathrm{~nm} \rightarrow$ $\mathrm{ChAB}-\mathrm{I}, 47 \mathrm{~nm} \rightarrow \mathrm{CQ}-2,30 \mathrm{~nm} \rightarrow \mathrm{PB}-3,28 \mathrm{~nm} \rightarrow$ $\mathrm{CQ}_{\mathrm{PB}}-3,45 \mathrm{~nm}$. Verifica-se que $\circ L_{\mathrm{c}}$ do coque é maior que $o$ do char e, portanto, possui uma estrutura cristalina mais organizada, o que está de acordo com a literatura. ${ }^{(3,9)}$ A fração $250-425 \mu \mathrm{m}$ do $\mathrm{PB}\left(\mathrm{CQ}_{\mathrm{PB}}\right)$ apresenta o maior valor de $L_{\text {c. }}$ Segundo Sahajwalla e Gupta ${ }^{(9)}$, as frações maiores que $250 \mu \mathrm{m}$ são compostas unicamente por coque. Segundo os autores, a fração $250-425 \mu \mathrm{m}$ do PB é a mais indicada para representar o padrão de coque, uma vez que esse material sofre grafitização no $\mathrm{AF}$, quando submetido a temperaturas superiores às que foi gerado. $O$ pó de balão apresenta $L_{c}$ próximo ao do coque, indicando a predominância de finos desse material em sua composição.

\subsubsection{Proporção de char e finos de coque nas amostras de pó de balão}

Os difratogramas das frações granulométricas do pó de balão PB são apresentados na Figura 3.

As diferenças nos perfis do pico (002) do carbono nas frações do PB resultam de diferentes tamanhos de cristalitos $\left(L_{c}\right)$; portanto, indicam diferentes composições de char e coque.

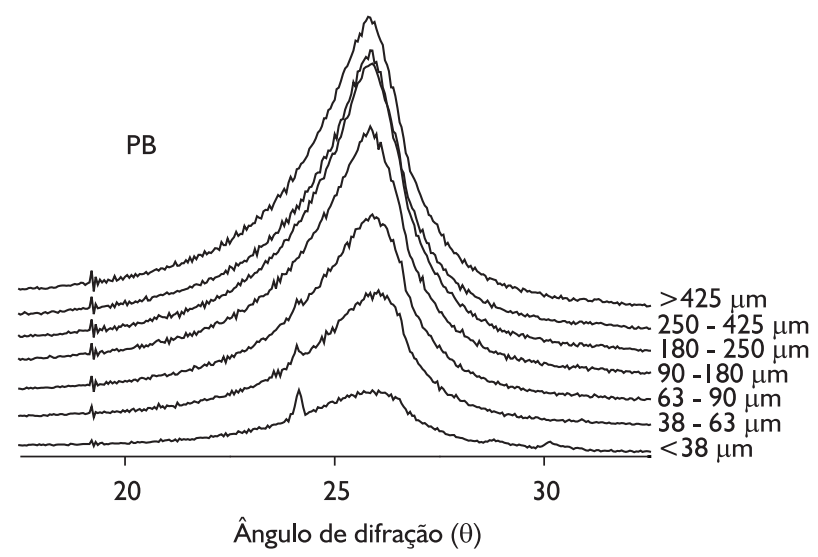

Figura 3. Espectro de raios $X$ e perfis do pico (002) do carbono para as frações granulométricas do $\mathrm{PB}\left(\mathrm{Cu} \mathrm{K} \alpha_{1}\right)$.
A Tabela 2 fornece os valores de $L_{c}$, carbono total e a composição char e coque para cada fração do PB. Os teores de char e coque são calculados a partir do valor de $L_{c}$ dos padrões $100 \%$ ChAB - I,47 nm e $100 \%$ de $\mathrm{CQ}_{\mathrm{PB}}-3,45 \mathrm{~nm}$. As frações superiores a $250 \mu \mathrm{m}$ são consideradas como compostas unicamente por coque.

Os teores totais de finos de coque e char no PB são, respectivamente, $37,45 \%$ e $2,95 \%$. Portanto, considerando somente a matéria carbonosa do $\mathrm{PB}$, cerca de $93 \%$ está na forma de coque e 7\%, na forma de char. Considerando estes resultados, os parâmetros operacionais do AF em estudo e um procedimento de análise semelhante, para amostras de lama, seria possível calcular o teor de char incombusto por tonelada de gusa produzido.

A fração 250 - $425 \mu \mathrm{m}$ apresenta maior valor de $L_{c}(3,45 \mathrm{~nm})$, sugerindo uma composição predominante de coque para esta faixa. A fração $<38 \mu \mathrm{m}$ apresenta - menor valor de $L_{c}(2,55 \mathrm{~nm})$, o que indica uma maior proporção de char. Apesar da literatura ${ }^{(9)}$ identificar que as frações menores que $63 \mu \mathrm{m}$ sejam compostas unicamente por char, para o pó em estudo, isto não pode ser confirmado, uma vez que estas amostras apresentaram valores de $L_{c}$ superiores ao char original $\left(L_{c} \quad I, 47 \mathrm{~nm}\right)$. Segundo Gupta et al. ${ }^{(4)}$, a fração $>425 \mu \mathrm{m}$, também considerada como composta apenas por coque, normalmente apresenta $L_{c}$ inferior ao da faixa $250-425 \mu \mathrm{m}$, indicando que sua estrutura se apresenta mais desorganizada, em relação à faixa mais fina. Acredita-se que essas amostras $(>425 \mu \mathrm{m})$ de finos de coque podem ter entrado em contato com a escória ou terem sofrido alguma gaseificação.

\section{CONCLUSÕES}

Com base nos resultados apresentados, pode-se concluir que:

- A técnica de DRX apresenta-se adequada para distinguir os materiais carbonosos presentes no pó de balão do AF. Com a sua utilização identifica-se que $\circ$ coque apresenta uma estrutura cristalina mais organizada que a do char, verificado pelo seu maior valor de $L_{c}$;

Tabela 2. Carbono total, $L_{c}$, e teor de char e coque para o PB, em diferentes frações granulométricas

\begin{tabular}{ccccc}
\hline $\begin{array}{c}\text { Fração granulométrica } \\
\text { do PB }(\mu \mathbf{m})\end{array}$ & $\begin{array}{c}\text { Carbono total no pó } \\
(\%)\end{array}$ & $\begin{array}{c}L_{c} \text {, estrutura carbonosa } \\
(\mathbf{n m})\end{array}$ & $\begin{array}{c}\text { Teor de char no pó } \\
(\%)\end{array}$ & $\begin{array}{c}\text { Teor de coque no pó } \\
(\%)\end{array}$ \\
\hline$<38$ & 0,08 & 2,55 & 0,04 & 0,04 \\
$38-63$ & 1,05 & 2,91 & 0,29 & 0,76 \\
$63-90$ & 1,98 & 2,93 & 0,52 & 1,46 \\
$88-180$ & 11,70 & 3,18 & 1,60 & 10,10 \\
$180-250$ & 12,62 & 3,37 & 0,52 & 12,10 \\
$250-425$ & 7,75 & 3,45 & - & 7,75 \\
$>425$ & 5,22 & 3,44 & - & 5,22 \\
$P B_{\text {total }}$ & 40,40 & 3,28 & 2,95 & 37,45 \\
\hline
\end{tabular}


- As frações granulométricas do pó de balão apresentam diferentes valores de $L_{c}$, indicando distintas composições de char e coque. A fração 250 - $425 \mu \mathrm{m}$ apresenta o maior valor de $L_{c}$, sugerindo uma composição exclusiva de coque para esta faixa, enquanto a fração $<38 \mu \mathrm{m}$ apresenta o menor valor de $L_{c}$, indicando uma predominância de char;

- A técnica utilizada permite a quantificação dos teores de char e coque presentes no pó de balão. Para o PB em estudo, verifica-se que cerca de $93 \%$ da matéria carbonosa está na forma de coque e $7 \%$, na forma de char;
- Com o uso combinado da técnica de DRX e análise elementar de carbono é possível estabelecer um procedimento analítico padrão para o monitoramento da composição de char e finos de coque no pó de balão do AF.

\section{Agradecimentos}

Os autores agradecem ao Conselho Nacional de Desenvolvimento Científico e Tecnológico - CNPq, pelo suporte financeiro; à Rede de Carvão e ao M.Sc., Eng. Henriquison M.B. Reis, da Usiminas, pela produção das amostras de char.

\section{REFERÊNCIAS}

I CARPENTER. A. M. Use of PCI in blast furnaces. London: IEA Clean Coal Centre, 2006. (CCC/I I6)

2 BABICH, A. et al Ironmaking. Aachen: Mainz GmbH, 2008.

3 SAHAJWALLA, V. et al Determination of proportions of coal char and coke fines in the off-gas blast furnace samples. In: IRONMAKING CONFERENCE, 59., 2000, Pittsburgh, USA. Proceedings... Warrendale: Iron and Steel Society, 2000. p. 305-II.

4 GUPTA, S. K. et al Atomic structure of coke fines in blast-furnace dust and their origin in operating blast furnaces. In: IRONMAKING CONFERENCE, 62., 2003, Indianápolis, Indiana. Proceedings... Warrendale: Iron and Steel Society, 2003. p. $84 \mathrm{I}-56$.

5 RIBAS, M. A. et al Identificação do char no pó de coletor do alto-forno através de técnicas de microscopia. In: $X X X$ SEMINÁRIO DE REDUÇÃO DE MINÉRIO DE FERRO, 30., 1999, Belo Horizonte. Anais... São Paulo: Associação Brasileira de Metalurgia e Materiais, 1999. p. 543-52.

6 YAMAGUCHI, K. et al Test on high-rate pulverized coal injection operation at Kimitsu n.3 blast furnace. ISIJ International, v. 35, n. 2, p. |48-55, Feb. 1995. http://dx.doi.org/10.2355/isijinternational.35.|48

$7 \mathrm{WU}, \mathrm{K}$. et al. Research on unconsumed fine coke and pulverized coal of bf dust under different $\mathrm{PCl}$ rates in $\mathrm{BF}$ at Capital Steel Co. ISIJ International, v. 50, n. 3, p. 390-5, Mar. 20 I0. http://dx.doi.org//0.2355/isijinternational.50.390

8 SAHAJWALLA, V. et al Combustion characteristics of pulverized coals and char released in blast furnace off-gas. In: IRONMAKING CONFERENCE, 62., 2003, Indianápolis, Indiana. Proceedings... Warrendale: Iron and Steel Society, 2003. p. 775-86.

9 SAHAJWALLA, V.; GUPTA S. PCI coal combustion behavior and residual coal char carryover in the blast furnaces of three American steel companies during pulverized coal injection (PCI) at high rates. Washington, DC: American Iron and Steel Institute, 2005.

I0 REIS, H. M. B. et al Influência do rank e da composição maceral de carvão pulverizado na eficiência de combustão no raceway do alto-forno. In: SEMINÁRIO DE REDUÇÃO DE MINÉRIO DE FERRO E MATÉRIAS-PRIMAS, 33.; SIMPÓSIO BRASILEIRO DE MINÉRIO DE FERRO, 4., 2003, Ouro Preto, MG, 2003. Anais... São Paulo: Associação Brasileira de Metalurgia e Materiais, 2003. p. 467-76.

I I AMERICAN SOCIETY FOR TESTING AND MATERIALS. ASTM D20I 3 - Standard practice for preparing coal samples for analysis. West Conshohocken, 2007.

I2 AMERICAN SOCIETY FOR TESTING AND MATERIALS. ASTM D5I42 - Standard test methods for proximate analysis of the analysis sample of coal and coke by instrumental procedures. West Conshohocken, 2009.

I 3 AMERICAN SOCIETY FOR TESTING AND MATERIALS. ASTM D4239 - Standard test methods for sulfur in the analysis sample of coal and coke using high-temperature tube furnace combustion methods. West Conshohocken, 2008.

I4 AMERICAN SOCIETY FOR TESTING AND MATERIALS. ASTM D5373 - Standard test methods for instrumental determination of carbon, hydrogen, and nitrogen in laboratory samples of coal. West Conshohocken, 2007. 
Machado et al.

I5 LU, L. et al. Quantitative X-ray diffraction Analysis and its application to various coals. Carbon, v. 39, n. I2, p. I82I-33, Oct. 200I. http://dx.doi.org/I0.1016/50008-6223(00)003I8-3

16 WARREN. B. E. X-ray diffraction random layer lattices. Physical Review, v. 59, n. 9, p 693-8, 194I. http://dx.doi. org/I0.I I03/PhysRev.59.693

Recebido em: $17 / 01 / 2011$

Aceito em: 17/08/20II 